\title{
Ground Moving Target Imaging (GMTIm) Algorithm in OFDM SAR Based on First-Order Keystone Transform
}

\author{
Mohammad Zeighami ${ }^{1, *}$, Mohammad Hassan Bastani' ${ }^{2}$, Fereidoun Behnia ${ }^{2}$ \\ ${ }^{1}$ Department of Electronic, Science and Research Branch, Islamic Azad University, Iran \\ ${ }^{2}$ Department Electrical Engineering, Sharif University of Technology, Tehran, Iran
}

Received October 21, 2019; Revised December 25, 2019; Accepted December 30, 2019

Copyright $\subseteq 2020$ by authors, all rights reserved. Authors agree that this article remains permanently open access under the terms of the Creative Commons Attribution License 4.0 International License

\begin{abstract}
The use of Orthogonal Frequency Division Multiplexing (OFDM) waveform in place of Linear Frequency Modulation (LFM) signal is one of the latest topics in the field of Synthetic Aperture Radar (SAR) imaging. The advantages of imaging by this technique include resistance to certain types of radar jamming techniques, reduced ambiguity in Doppler spectrum with an increase in the number of sub-bands, and extraction of target phase history based on phase approximation of all OFDM subcarriers. In conventional SAR imaging algorithms, it is assumed that the targets are stationary in the imaging zone as these algorithms do not have the required efficiency to image moving targets, leading to blurred images and high Integrated Side Lobe Ratio (ISLR). To circumvent these problems, there is a requirement of designing appropriate imaging algorithms, keeping in mind the important issues related to the moving targets in the imaging zone. Thus, in this study, we propose the implementation of the OFDM waveform for imaging moving targets alongside the first-order keystone transform to solve the above-mentioned problems.
\end{abstract}

Keywords OFDM SAR, GMTIm, First-Order Keystone Transform

\section{Introduction}

$\mathrm{SAR}^{1}$ is a microwave imaging system that can take 2-D images from a given scene, under all climatic conditions. "Range", which measures the line of sight distance from the radar to the target, is one of the dimensions of this

\footnotetext{
${ }^{1}$ Synthetic Aperture Radar
}

imaging system. Another dimension of the imaging system is the "Azimuth", which is perpendicular to the range axis. Range is measured using pulse compression techniques and is based on the accurate measurement of the time elapsed from the moment the pulse is transmitted to the moment it returns from the target [1]. With the movement of the SAR radar, pulses are continuously sent, and the returning echoes are received and saved in an echo storage memory. Since the radar moves with respect to the ground, the returned echoes are shifted in frequency (known as Doppler shift) with respect to the transmitted signals. The comparison of the Doppler shifted signals with a reference frequency signal enables the returned signals to be focused on a single point, resulting in an effective increase of the antenna length.

In addition, $\mathrm{OFD}^{2}$ is widely used in telecommunication systems [2-4]. Due to the orthogonal property of the signal, it is possible to modulate independent data streams on each of the subcarriers without creating any interference. Given these unique properties, the OFDM waveform has been highly investigated by many researchers in the SAR field during recent years [5]. A previous study examined the simultaneous implementation of radar and data communication capabilities in a single system using OFDM [6]. This system was reportedly implemented on $\mathrm{SDR}^{3}$ hardware and could achieve a bit rate of $57 \mathrm{Mb} / \mathrm{s}$ with a bit error rate of less than 5\%. A high-resolution SAR imaging system based on space-time coding $\mathrm{MIMO}^{4}$ and OFDM was proposed in another study [7]. This system used MIMO configuration in the elevation direction, space time coding in the azimuth direction, and employed OFDM

\footnotetext{
2 Orthogonal Frequency Division Multiplexing

3 Software Defined Radio

${ }^{4}$ Multi-Input Multi-Output
} 
waveform diversity.

In SAR imaging algorithms, the targets are assumed to be stationary in the imaging zone. This is because the algorithms mentioned above will not be properly applicable with moving targets as it would result in opaque and blurred images. Thus, it is necessary to redesign the imaging algorithms and devise appropriate filters and functions, considering the presence of moving targets. We intend to use the keystone transform in our moving target imaging scheme. A previous study [8] has implemented the GMTIm $^{5}$ Algorithm by means of first-order keystone transform. Applying interpolation kernels is the first step for implementing keystone transform. This method, however, is not usually used because of its very high computational load. Thus, the chirp scaling technique has been proposed for implementing keystone transform [9]. In this method, the idea of stationary phase without resorting to interpolation kernels is implemented along with their heavy computational loads.

In the present study, OFDM is briefly reviewed, and then its applications in SAR imaging of moving targets are discussed. In this regard, the imaging geometry is drawn in the presence of moving targets, and the instantaneous range equation is extracted for use in the processing algorithm. The imaging process is expressed in range and azimuth within the framework of the first-order keystone transform. In the end, the results of the simulation are shown for a point target in each of the processing phases.

\section{Modeling Ultra-Wideband Communication (UWB) OFDM Waveform}

The commonly used OFDM waveform in telecommunication digital systems is described below:

$$
s_{a}(t)=\sum_{k=1}^{N} x(k) \exp \left(j\left(2 \pi k t / T_{p}+\phi_{0}(t)\right)\right), 0 \prec t \prec T_{p}
$$

In this equation, $x(k)$ denotes $k^{\text {th }}$ data symbol from the data vector $x=[x(1) x(2) \ldots x(N)], N$ is the number of subcarriers, and $T_{p}$ indicates pulse transmission period. The $k^{\text {th }}$ subcarrier belongs to the sub-band $k \Delta f$ with $\Delta f=1 / T_{p}$ being equal to the bandwidth of any of the sub-bands. Although these sub-bands overlap, the peak of each subband is placed on the null point of another subband due to the orthogonal property of the waveform. If the real part of Eq. (1) was considered with the value of $\phi_{0}(t)=0$, we would have:

$$
s(t)=\sum_{k=1}^{N} x(k) \cos (2 \pi \Delta f k t), 0 \prec t \prec T_{p}
$$

The above equation indicates the waveform used in practical radar systems, and it is utilized as the reference signal in the following discussion. It is clear from Eqn. 2 that $s(t)$ is the sum of sinusoidal pulses with a transmission period of $T_{p}$. Signal Spectrum $S(f)$ is obtained by applying Fourier Transform to signal $s_{a}(t)$ :

$$
\begin{aligned}
& S(f) \\
& =F_{(t)}\left[S_{a}(t)\right]=\sum_{k=1}^{N} x(k) \int_{-T_{p} / 2}^{T_{p} / 2} e^{j 2 \pi k \Delta f t} e^{-j \omega t} d t \\
& =\sum_{k=1}^{N} x(k) \frac{\sin \left(\pi(f-k \Delta f) T_{p}\right)}{\pi(f-k \Delta f) T_{p}}
\end{aligned}
$$

The $k^{\text {th }}$ Sinc function is related to the $k^{\text {th }}$ sub-band center, and these centers are placed in frequency intervals of $k \Delta f$. The signal spectrum $S(f)$ along with the $N$ subcarriers is shown in Figure 1.

\footnotetext{
${ }^{5}$ Ground Moving Target Imaging
} 


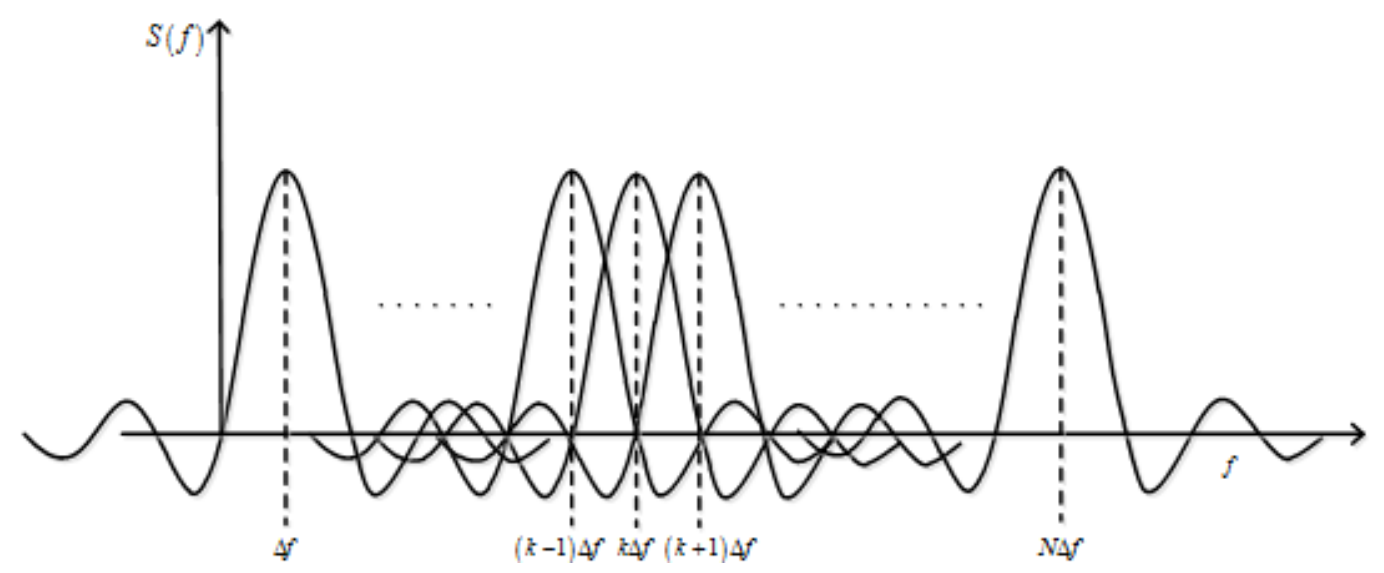

Figure 1. OFDM Spectrum Signal with N Subcarriers

As shown in Figure 1, the width of the main lobe of each Sinc function is $2 \Delta f$, and since $\Delta f=1 / T_{p}$, the width of the main lobe depends on the period of pulse transmission only. When the total bandwidth is fixed, the number of sub-bands will be proportional to the period of pulse transmission, i.e., an increase in the number of sub-bands will result in a corresponding increase in the pulse transmission period.

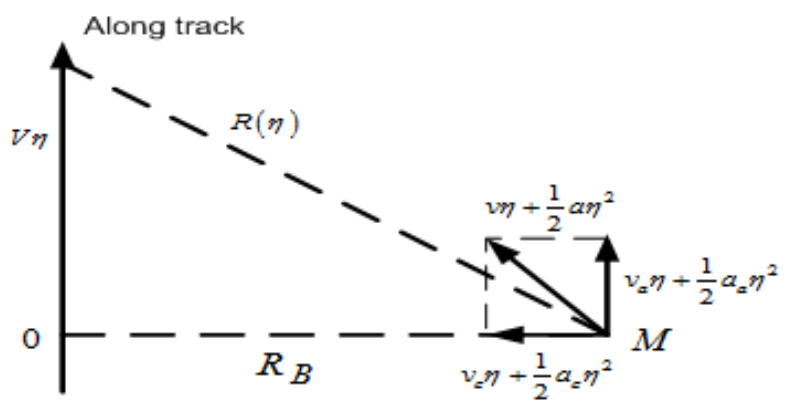

Figure 2. Geometry of Imaging Moving Targets

\section{Geometry of Moving Targets Imaging in SAR}

In Figure 2, a simplified geometry of SAR imaging has been displayed with zero-laevorotation (the beam is placed on broadside). In this figure, $V$ denotes platform speed, $M$ is the moving target, and finally, $v_{a}$ and $a_{a}$ indicate velocity and acceleration of the moving target $\mathrm{M}$ along an axis parallel to the platform path. Similarly, $v_{c}$ and $a_{c}$ denote velocity and acceleration of the moving target along a perpendicular axis to the platform's path. Likewise, $R_{B}$ is the shortest distance between the radar and the target, and $\eta$ is the slow time. If the target moves toward the radar, $V_{c}$ is positive. The instantaneous range between the radar and the moving target is expressed by means of the first order Taylor expansion as follows:

$$
\begin{aligned}
& R(\eta)=\sqrt{\left(V \eta-v_{a} \eta-\frac{1}{2} a_{a} \eta^{2}\right)^{2}+\left(R_{B}-v_{c} \eta-\frac{1}{2} a_{c} \eta^{2}\right)^{2}} \\
& \cong R_{B}-v_{c} \eta+\frac{\left(V-v_{a}\right)^{2}-a_{c} R_{B}}{2 R_{B}} \eta^{2}
\end{aligned}
$$

\section{Proposed OFDM SAR Processing Algorithm for Imaging of Moving Targets}

In previous sections, the required background was presented for OFDM, and the geometry for imaging was also extracted to derive the equation of instantaneous range in the processing algorithm. These issues are used in this section, and the imaging algorithm in SAR is proposed within the framework of OFDM waveforms for imaging of moving targets.

\subsection{The Transmitted OFDM Signal}

With minor modification of the equation presented earlier, the transmitted OFDM signal in a single period and used in this application can be written as:

$$
s_{t}(\tau)=\operatorname{rect}\left(\frac{\tau}{T_{p}}\right) \sum_{k=1}^{N} x(k) \exp \left(j\left(2 \pi\left(f_{0}+k \Delta f\right) \tau\right)\right)
$$

Here, $\tau$ is the fast time, $f_{0}$ is the carrier frequency, and rect function is the transmitted pulse envelope. During the reception interval, the radar receiver waits for receiving the returning signal from the target. The range of the returned signal is one dimension of the signal space and a function of the fast time. A simplified view of the transmitted and received waveforms is depicted in Figure 3. 


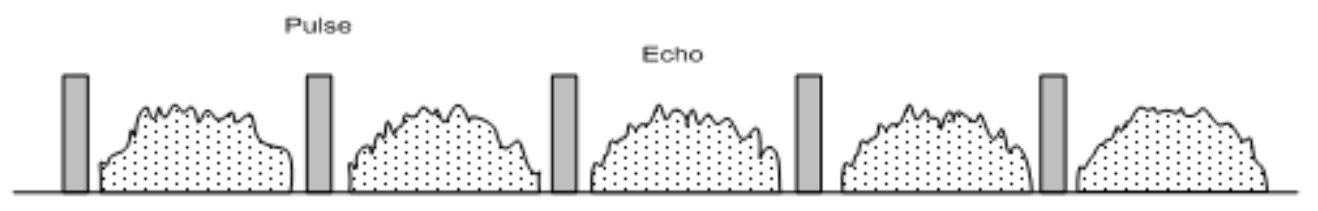

Figure 3. The Reception and Transmission of a Signal in SAR Radar

\subsection{Received OFDM Signal from the Moving Target}

The received SAR signal, which is basically the delayed version of the signal in Equation 5, can be written as follows:

$$
s_{r}(\tau, \eta)=\sigma_{0} \sum_{k=1}^{N} \operatorname{rect}\left(\frac{\tau-\frac{2 R(\eta)}{c}}{T_{p}}\right) \operatorname{rect}\left(\frac{\eta}{L /\left(V-v_{a}\right)}\right) x(k) \exp \left(j\left(2 \pi\left(f_{0}+k \Delta f\right)\left(\tau-\frac{2 R(\eta)}{c}\right)\right)\right)
$$

One dimension of this 2-D signal is the range-time dimension represented by $\tau$, and the other dimension is the slow time dimension represented by $\eta$. In Equation 6, $\sigma_{0}$ denotes radar cross-section, $c$ is the speed of propagation, $T_{p}$ is the pulse transmission period, $L$ indicates the synthetic aperture, rect $(\eta)$ stands for antenna array size in the azimuth direction, $\operatorname{rect}(\tau)$ is OFDM signal envelope, and $\frac{2 R(\eta)}{c}$ is the delay due to signal reflection from the target. In This equation the attenuation due to signal propagation hasn't been considered. The value of $R(\eta)$ is calculated for the moving target by means of Equation 4. After translation to baseband, the received signal of Equation 6 changes to the following form:

$$
s_{r 1}(\tau, \eta)=\sigma_{0} \sum_{k=1}^{N} r e c t\left(\frac{\tau-\frac{2 R(\eta)}{c}}{T_{p}}\right) \operatorname{rect}\left(\frac{\eta}{L /\left(V-v_{a}\right)}\right) x(k) \exp \left(-j 4 \pi f_{0} \frac{R(\eta)}{c}\right) \exp \left(j 2 \pi k \Delta f\left(\tau-\frac{2 R(\eta)}{c}\right)\right)
$$

\subsection{Compression in Range}

Signal compression in the range direction is the first step in SAR processing algorithms. For this purpose, the Fourier Transform of the signal is first calculated. This transform naturally applies to the part of Equation 7 that depends on $\tau$. The result is depicted below:

$$
\mathcal{F}_{\tau}\left\{\sum_{k=1}^{N} r e c t\left(\frac{\tau-\frac{2 R(\eta)}{c}}{T_{p}}\right) x(k) \exp \left(j 2 \pi k \Delta f\left(\tau-\frac{2 R(\eta)}{c}\right)\right)\right\}=\sum_{k=1}^{N} x(k) \frac{\sin \left(\pi\left(f_{\tau}-k \Delta f\right) T_{p}\right)}{\pi\left(f_{\tau}-k \Delta f\right) T_{p}} \exp \left(-j \frac{4 \pi f_{\tau} R(\eta)}{c}\right)
$$

The match filter in the range direction is equal to the Fourier Transform of the transmitted OFDM signal, which is calculated as follows:

$$
H_{r c}\left(f_{\tau}\right)=\left[\mathcal{F}_{\tau}\left\{\sum_{k=1}^{N} \operatorname{rect}\left(\frac{\tau}{T_{p}}\right) x(k) \exp (j 2 \pi k \Delta f \tau)\right\}\right]^{*}=\sum_{k=1}^{N} x(k) \frac{\sin \left(\pi\left(f_{\tau}-k \Delta f\right) T_{p}\right)}{\pi\left(f_{\tau}-k \Delta f\right) T_{p}}
$$


This spectrum was shown in Figure 1. As can be seen, with increasing number of sub-bands, the spectrum changes to a rectangular pulse form in the frequency domain, and its envelope can be approximated with a rectangular function. The IFT ${ }^{6}$ of a rectangular function is a Sinc function; hence, by substituting Equation 8 in Fourier Transform of Equation 7, we have:

$$
\begin{aligned}
& S_{r 2}\left(f_{\tau}, \eta\right) \\
& =\sigma_{0} \sum_{k=1}^{N} r e c t\left(\frac{\eta}{L /\left(V-v_{a}\right)}\right) \\
& \times \exp \left(-j 4 \pi\left(f_{0}+f_{\tau}\right) \frac{R(\eta)}{c}\right) \\
& \times x(k) \times \frac{\sin \left(\pi\left(f_{\tau}-k \Delta f\right) T_{p}\right)}{\pi\left(f_{\tau}-k \Delta f\right) T_{p}}
\end{aligned}
$$

Multiplying Equation 9 with Equation 10 and using the new definition of

$$
W\left(f_{\tau}\right)=\left(\sum_{k=1}^{N} x(k) \frac{\sin \left(\pi\left(f_{\tau}-k \Delta f\right) T_{p}\right)}{\pi\left(f_{\tau}-k \Delta f\right) T_{p}}\right)^{2}, \quad \text { we will }
$$

have:

$$
\begin{aligned}
& S_{r 3}\left(f_{\tau}, \eta\right)=\sigma_{0} W\left(f_{\tau}\right) \times r e c t\left(\frac{\eta}{L /\left(V-v_{a}\right)}\right) \\
& \times \exp \left(-j 4 \pi\left(f_{0}+f_{\tau}\right) \frac{R(\eta)}{c}\right)
\end{aligned}
$$

By increasing the number of OFDM subcarriers, the envelope of this spectrum approaches the rectangular form (i.e., rect $\left(\frac{f_{\tau}}{N \Delta f}\right)$ ) as stated before. Thus, by taking the inverse Fourier Transform of Equation 11 with respect to range, a Sinc function is created whose peak location corresponds to OFDM signal transmit time for any specific value of azimuth and any point target position. Meanwhile, it may be inferred from Equation 11 that the range has been coupled with azimuth. On substituting Equation 4 in Equation 11, the expression for the phase will be given as Equation 12 below:

$$
\begin{aligned}
& \phi_{r c}\left(f_{\tau}, \eta\right) \\
& =-\frac{4 \pi\left(f_{0}+f_{\tau}\right)}{c} R_{B}+\frac{4 \pi\left(f_{0}+f_{\tau}\right)}{c} v_{c} \eta \\
& -\frac{2 \pi\left(f_{0}+f_{\tau}\right)}{c} \cdot \frac{\left(V-v_{a}\right)^{2}-a_{c} R_{B}}{R_{B}} \eta^{2}
\end{aligned}
$$

\footnotetext{
${ }^{6}$ Inverse Fourier Transform
}

In Equation 12, the first term remains constant and does not affect the rest of the processing. The second term is related to the displacement of range (Range walk) and Doppler shift, which can be compensated knowing the speed of the target perpendicular to its moving path of radar. The third term corresponds to the range curvature and Doppler frequency modulation that can also be compensated knowing the velocity of the target along the moving path of radar and acceleration of the target perpendicular to the moving path of radar. In practice, since the moving targets do not move in groups, the target signal is hidden by clutter. For this reason, a high-resolution imaging system is required; these systems spend a lot of time collecting data (larger synthetic aperture) leading to greater range curvature.

In this case, curvature lines from various targets overlap after compression operation in range, separating targets from each other and approximating their parameters that get very difficult. To solve this problem, two algorithms have previously been purposed, namely the second-order and the first-order keystone transforms.

The aim of using keystone transform in both the techniques is for correcting the Range Curvature and Range Walk. However, previous works have reported that the second-order keystone transform is not optimal [8] as it needs a lot of computational tasks that must be carried out for each moving target. Comparatively, this problem does not exist in the first-order keystone transform. The second-order keystone transform method needs to estimate the Doppler centroid for every range frequency before any range migration correction. Therefore, it is not computationally efficient and requires a high $\mathrm{SCNR}^{7}$. Comparatively, in the first-order keystone transform, the imaging of moving targets is performed with large range walk and curvature, and the Doppler centroid is estimated after range walk correction. Therefore, the first-order keystone transform is employed in our study to form the image of the moving target.

The processing phases of GMTIm Algorithm based on the first-order keystone transform are depicted in Figure 4. In this algorithm, initially the range walk is compensated, leading to correction of the range curve rotation (Range Walk) due to the motion-related parameters of the targets. Then, the range curvature is compensated, and the curvature caused by $\mathrm{RCM}^{8}$ as well as the target movement parameters are corrected. Eventually, the appropriate match filter in the azimuth direction is applied by means of Azimuth $\mathrm{FM}^{9}$ ratio.

\footnotetext{
${ }^{7}$ Signal Clutter Noise Ratio

${ }^{8}$ Range Cell Migration

9 Frequency Modulation
} 


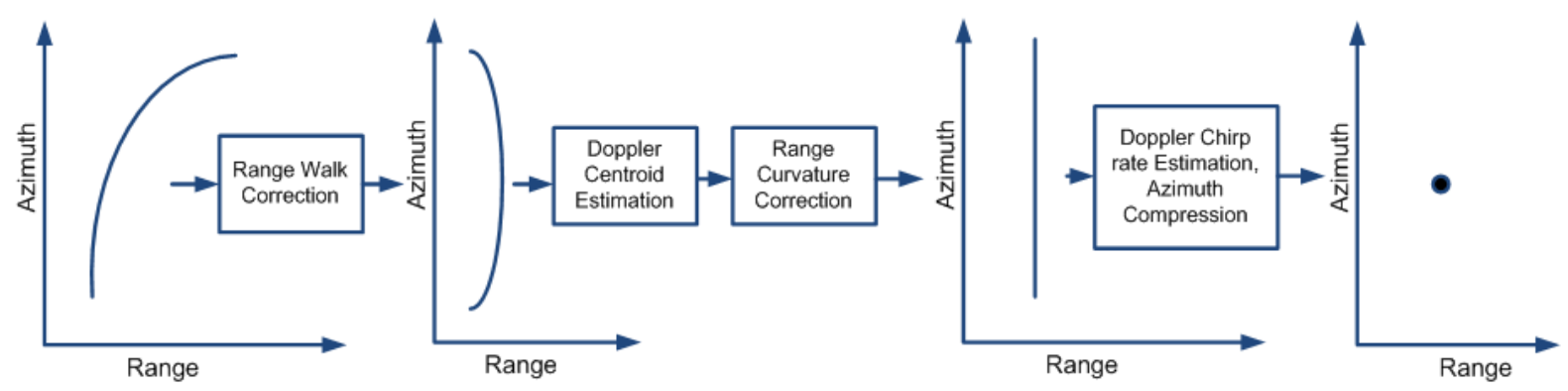

Figure 4. Processing Steps by means of First-Order Keystone Transform

The curvature rotation (Range Walk) of the moving targets can be compensated by means of first-order keystone transform by scaling azimuth time vector for any value of range frequency such that:

$$
\eta=\left(\frac{f_{c}}{f_{c}+f_{\tau}}\right) \eta_{m}
$$

In this equation, $\eta_{m}$ is the new slow time after the first-order keystone is implemented [10]. The implementation of Equation 13 using interpolation techniques requires a lot of computational loads; as a result, the chirp scaling method [9] is employed given that the $\mathrm{TBP}^{10}$ value is higher than 200 . The summary of this technique is presented in the next section.

\subsection{Range Walk Correction by Means of First-Order Keystone Transform}

On substituting Eqn. 13 in Eqn. 12, we have:

$$
\begin{aligned}
& \varphi_{r c}\left(f_{\tau}, \eta_{m}\right)=-\frac{4 \pi\left(f_{c}+f_{\tau}\right)}{c} R_{B}+\frac{4 \pi}{\lambda} v_{c} \eta_{m} \\
& -\frac{2 \pi}{c} \cdot \frac{\left(V-v_{a}\right)^{2}-a_{c} R_{B}}{R_{B}} \frac{f_{c}{ }^{2}}{\left(f_{c}+f_{\tau}\right)} \eta_{m}{ }^{2}
\end{aligned}
$$

The linear dependency of Range Walk (second term in eq.12) on the range frequency has been removed due to the use of first-order keystone transform. In the last part of Equation 14, however, a type of quadratic dependency on the range frequency has been created, which is known as Range Curvature (residual coupling is present in the quadratic term, which corresponds to the range curvature). This part represents RCM curvature, which can be compensated by correction of the filter in 2-D frequency domain.

The second term in Equation 14 represents Doppler centroid frequency $\left(f_{d c}\right)$, which is calculated and compensated before range curvature correction operation by the pertinent techniques in Doppler approximation. This parameter can be computed without any sensitivity to Range Curvature by means of central Doppler approximation methods based on signal range information.

$$
f_{d c}=\frac{2 v_{c}}{\lambda}
$$

Similarly, the azimuth FM ratio in Equation 14 is presented as follows:

$$
K_{a}=2 \frac{\left(V-v_{a}\right)^{2}-a_{c} R_{B}}{\lambda R_{B}}
$$

After Doppler central frequency value correction, Equation 14 may be rewritten as follows:

$$
\begin{aligned}
& \varphi_{r c}\left(f_{\tau}, \eta_{m}\right)=-\frac{4 \pi\left(f_{c}+f_{\tau}\right)}{c} R_{B} \\
& -\frac{2 \pi}{c} \cdot \frac{\left(V-v_{a}\right)^{2}-a_{c} R_{B}}{R_{B}} \frac{f_{c}{ }^{2}}{\left(f_{c}+f_{\tau}\right)} \eta_{m}{ }^{2}
\end{aligned}
$$

Let us introduce the parameter $\mathrm{B}$ as follows:

$$
B=\frac{1}{c} \cdot \frac{\left(V-v_{a}\right)^{2}-a_{c} R_{B}}{R_{B}} \frac{f_{c}^{2}}{\left(f_{c}+f_{\tau}\right)}
$$

where, this parameter is related to the previously-introduced parameter $K_{a}$.

$$
B=\frac{K_{a}}{2} \frac{f_{c}}{\left(f_{c}+f_{\tau}\right)}
$$

Inserting parameter B in Eqn. 17 leads to:

$$
\varphi_{r c}\left(f_{\tau}, \eta_{m}\right)=-\frac{4 \pi\left(f_{c}+f_{\tau}\right)}{c} R_{B}-2 \pi B \eta_{m}{ }^{2}
$$

So, after Range Walk cancellation, Doppler centroid frequency correction, and rearrangement of parameters, the output of Equation 11 will be as follows:

$$
\begin{aligned}
& s_{r 4}\left(f_{\tau}, \eta_{m}\right)=\sigma_{0} \operatorname{rect}\left(\frac{f_{\tau}}{N \Delta f}\right) \\
& \times \operatorname{rect}\left(\frac{\eta_{m}}{L /\left(V-v_{a}\right)}\right) \exp \left(-j\left(\frac{4 \pi\left(f_{c}+f_{\tau}\right)}{c} R_{B}+2 \pi B \eta_{m}^{2}\right)\right)
\end{aligned}
$$

\subsection{Range Curvature Correction}

The Fourier Transform of azimuth signal can be derived 
by means of the POSP $^{11}$ technique from Equation 21 and can be presented as:

$$
S_{d c c}\left(f_{\tau}, f_{d}\right)=\int_{-\infty}^{\infty} s_{r 4}\left(f_{\tau}, \eta_{m}\right) \exp \left(-j 2 \pi f_{d} \eta_{m}\right) d \eta_{m}
$$

The phase function under this integral is derived by means of Equation 21 as below:

$$
\varphi_{d c c}\left(\eta_{m}\right)=-\frac{4 \pi\left(f_{c}+f_{\tau}\right)}{c} R_{B}-2 \pi B \eta_{m}{ }^{2}-2 \pi f_{d} \eta_{m}
$$

The derivative of $\varphi_{\text {dcc }}\left(\eta_{m}\right)$ with respect to $\eta_{m}$ is easily derived as follows:

$$
\frac{d \varphi_{d c c}\left(\eta_{m}\right)}{d \eta_{m}}=-4 \pi B \eta_{m}-2 \pi f_{d}
$$

To implement POSP, the range time for which that derivative equals to zero in Equation 24 should be calculated as follows:

$$
\eta_{m}=-\frac{f_{d}}{2 B}
$$

On substituting Equation 25 in Equation 23, the phase in 2-D Fourier Transform term will be as below:

$$
\varphi_{d c c}\left(f_{\tau}, f_{d}\right)=-\frac{4 \pi\left(f_{c}+f_{\tau}\right)}{c} R_{B}+\frac{\pi f_{d}^{2}}{2 B}
$$

Likewise, the above formula may be modified to include azimuth FM ratio $\left(K_{a}\right)$ :

$$
\varphi_{d c c}\left(f_{\tau}, f_{d}\right)=-\frac{4 \pi\left(f_{c}+f_{\tau}\right)}{c} R_{B}+\frac{\pi}{K_{a}} \frac{f_{c}+f_{\tau}}{f_{c}} f_{d}^{2}
$$

Based on these explanations, the rate of range curvature in 2-D frequency domain may be expressed as below:

$$
\Delta R W_{2 d}=\frac{\pi}{K_{a}} \frac{f_{\tau}}{f_{c}} f_{d}^{2}
$$

Therefore, the required filter phase for range curvature effect correction will be of the form:

$$
H_{2 d}=-j \Delta R W_{2 d}
$$

It should be noted that $K_{a}$ varies for different targets with different ranges $\left(R_{B}\right)$ and velocities; as a result, an approximate value is used for ease of use. For example, in Equation 16, $V_{a}$ is much smaller than $V$ and at the same time the amount of acceleration along the moving path $\left(a_{c}\right)$ is also very small or even equal to zero. Likewise, the amount of range walk $R_{B}$ of the target can often be ignored with respect to the sweep width, and it is considered equal to the center of sweep width $R_{0}$. Given

\footnotetext{
${ }^{11}$ Principle of Stationary Phase
}

these points, the approximate value of $K_{a}$ in Eqn. 16 will be as follows:

$$
K_{a} \approx K_{a p}=\frac{2 V^{2} f_{c}}{c R_{0}}
$$

The correction filter for correction of curvature effect of Range walk will be as below:

$$
H_{2 d}=-j \frac{\pi c R_{0} f_{\tau} f_{d}^{2}}{2 V^{2} f_{c}^{2}}
$$

The above approximation may not always meet the requirements, and more accurate values for $K_{a}$ can be derived by means of analytic time-frequency techniques like Fast Fourier Transform or Wigner-Ville (quasi-probability) distribution.

Given these remarks, the result obtained after applying the filter in Equation 29 to the phase of Equation 27 can be expressed as follows:

$$
\varphi_{d c c}\left(f_{\tau}, f_{d}\right)=-\frac{4 \pi\left(f_{c}+f_{\tau}\right)}{c} R_{B}+\frac{\pi}{K_{a}} f_{d}^{2}
$$

The resulting signal will be of the form as given below:

$$
\begin{aligned}
& S_{R W C}\left(f_{\tau}, f_{d}\right)=W_{r}\left(f_{\tau}\right) \times W_{a}\left(f_{d}\right) \\
& \times \exp \left\{-j \frac{4 \pi\left(f_{c}+f_{\tau}\right)}{c} R_{B}+j \frac{\pi}{K_{a}} f_{d}{ }^{2}\right\}
\end{aligned}
$$

Applying inverse Fourier Transform in the range by means of time displacement property and phase multiplication of Fourier Transform, we have:

$$
\begin{aligned}
& S_{R W C}\left(\tau, f_{d}\right)=p_{r}\left(\tau-\frac{2 R_{B}}{c}\right) \times W_{a}\left(f_{d}\right) \\
& \times \exp \left\{-j \frac{4 \pi f_{c} R_{B}}{c}+j \frac{\pi}{K_{a}} f_{d}^{2}\right\}
\end{aligned}
$$

Here, the term $\exp \left\{-j \frac{4 \pi f_{c} R_{B}}{c}\right\}$ is a constant phase that can be ignored, and the equation can be rewritten as follows:

$$
S_{R W C}\left(\tau, f_{d}\right)=p_{r}\left(\tau-\frac{2 R_{B}}{c}\right) \times W_{a}\left(f_{d}\right) \times \exp \left\{j \frac{\pi}{K_{a}} f_{d}{ }^{2}\right\}
$$

\subsection{Compression in Azimuth}

The reference signal for the Doppler matched filter can be written as below:

$$
H_{a z}=\exp \left\{-j \frac{\pi}{K_{a}} f_{d}^{2}\right\}
$$

Multiplying Equation 36 by Equation 35 and applying inverse Fourier Transform, we will have:

$$
S_{A C}\left(\hat{t}, \tau_{m}\right)=p_{r}\left(\hat{t}-\frac{2 R_{B}}{c}\right) \times p_{a}\left(\tau_{m}\right)
$$




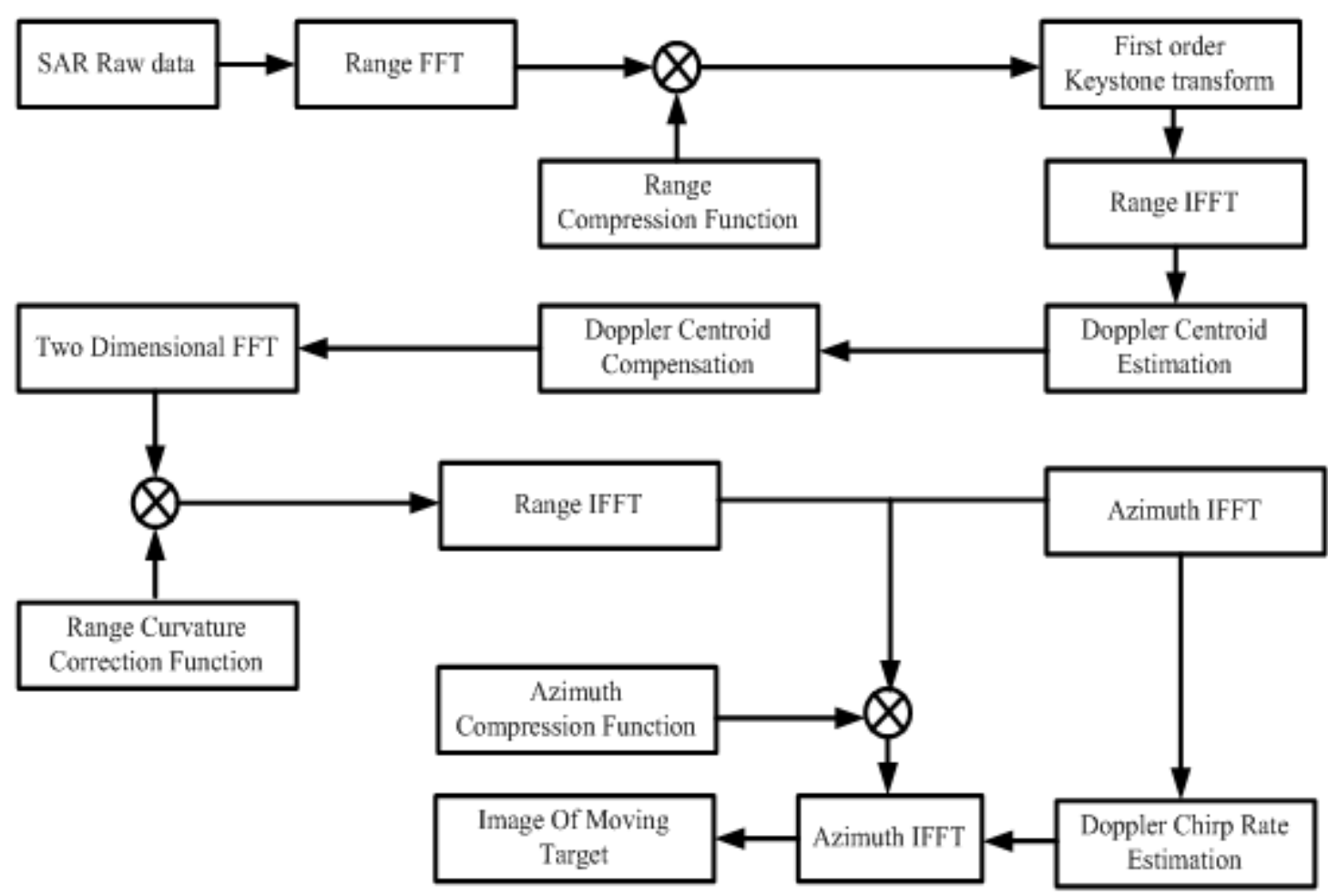

Figure 5. The Algorithm Flowchart for SAR Moving Target Image Formation

The summary of the presented algorithm, up to this point, is shown in Figure 5.

At this point, the process of forming an OFDM SAR image for the moving target gets completed. The implementation of the first-order keystone transform by means of chip scaling will be discussed in the next section.

\section{Implementation of First-Order Keystone Transform by Means of Chirp Scaling}

As noted in the previous section, the phase history linear term (Range Walk) of moving targets can be omitted using the first-order keystone transform. The linear term in the phase history (Range Walk) causes rotation of the range curvature, and if this term is deleted before the 2-D Fourier Transform, the second-order term of the phase history may be deleted by means of an appropriate correction filter in the 2-D frequency domain.

The question that may be raised here is how to implement the keystone transform. To the best of our knowledge, there are two general techniques for implementation of this transform: one technique is by employing interpolation methods and the other one is based on chirp scaling techniques. As mentioned in a previous study [9], however, the implementation of interpolation method in practical applications has been avoided due to its higher computational loads, and therefore, the chirp scaling method is suggested for this purpose. The chirp scaling technique is based on $\mathrm{RCMC}^{12}$ operation without using interpolation kernels [11], which results in increased speed for RCMC implementation. Since the stationary phase assumption will be used in POSP Fourier Transform computation to implement this technique, it requires TBP to be higher (i.e., more than 200) along the azimuth direction. Given the time of synthetic aperture formation in seconds in SAR and Doppler bandwidth of a few kilo Hertz, the time bandwidth product is greater than the minimum value, and so POSP technique can be utilized for chirp scaling. We will, thus, discuss the chirp scaling technique based on the POSP method, followed by the discussion on keystone transform.

The complete block diagram of chirp scaling technique is depicted in Figure 5. As already mentioned, the stationary Fourier Transform [9] is employed, and $\alpha+\gamma=\beta$ is the only condition that should be considered in this technique.

${ }^{12}$ Range Cell Migration Correction 


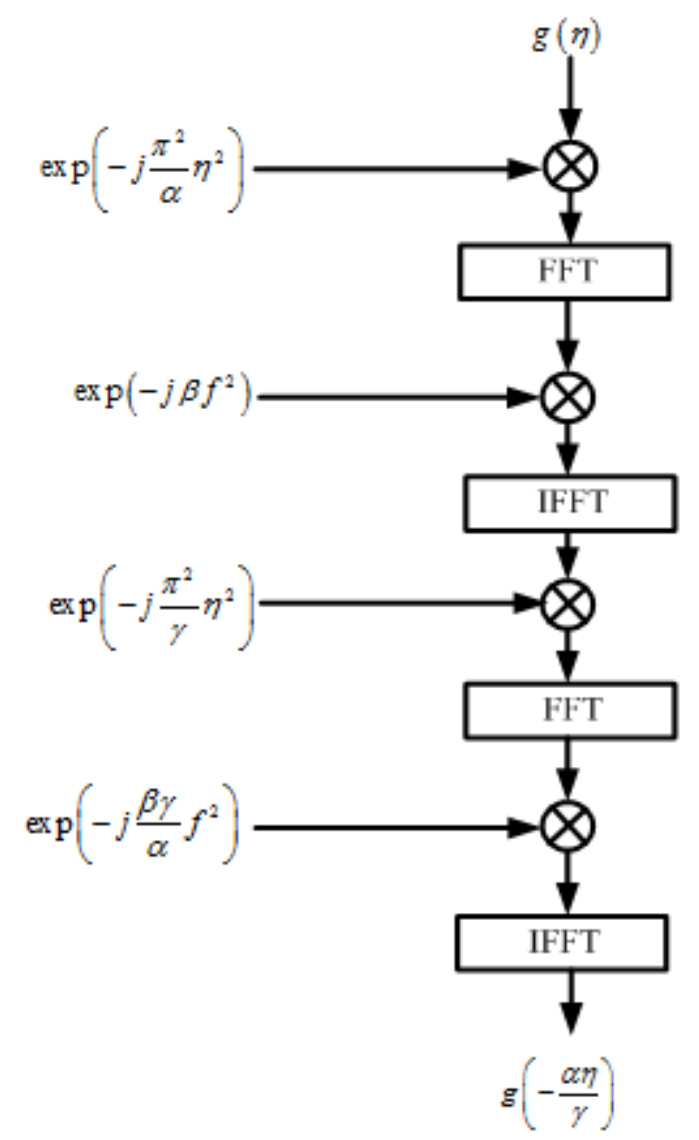

Figure 6. The Implementation Method of Chirp Scaling in the Frequency Field

In this figure, the signal $g(\eta)$ is converted into $g\left(-\frac{\alpha \eta}{\gamma}\right)$ by means of FFT $^{13}$ and IFFT $^{14}$ transforms and multiplication in the given phase functions. In Section 4-3, first-order keystone transform is derived to compensate for Range Walk, using this property. The received signal formula of the moving target after compression in the range (Equation 11) may be rewritten as follows:

$$
S_{r c}\left(f_{\tau}, \eta\right)=P\left(f_{\tau}\right) \times \exp \left(j\left(-\frac{4 \pi\left(f_{0}+f_{\tau}\right)}{c} R_{B}+\frac{4 \pi\left(f_{0}+f_{\tau}\right)}{c} v_{c} \eta-\frac{2 \pi\left(f_{0}+f_{\tau}\right)}{c} \cdot \frac{\left(V-v_{a}\right)^{2}-a_{c} R_{B}}{R_{B}} \eta^{2}\right)\right)
$$

where, $P\left(f_{\tau}\right)$ is considered as the signal envelope function in the frequency domain. In addition, the first-order keystone transform, which compensates for the rotation in curvature of the moving targets (Range Walk), is given below: Substituting Equation 39 in the phase of Equation 38, we will have:

$$
\eta=\left(\frac{f_{c}}{f_{c}+f_{\tau}}\right) \eta_{m}
$$

Substituting Equation 39 in the phase of Equation 38, we will have:

$$
S_{r c}\left(f_{\tau}, \eta_{m}\right)=P\left(f_{\tau}\right) \times \exp \left[j\left(-\frac{4 \pi\left(f_{c}+f_{\tau}\right)}{c} R_{B}+\frac{4 \pi}{\lambda} v_{c} \eta_{m}-\frac{2 \pi}{c} \cdot \frac{\left(V-v_{a}\right)^{2}-a_{c} R_{B}}{R_{B}} \frac{f_{c}^{2}}{\left(f_{c}+f_{\tau}\right)} \eta_{m}{ }^{2}\right)\right]
$$

\footnotetext{
${ }^{13}$ Fast Fourier Transform

${ }^{14}$ Inverse Fast Fourier Transf
} 
In fact, the principles of chirp scaling technique are employed in order to determine parameters $\alpha, \beta$, and $\gamma$ according to the block diagram of Figure 6 such that Equation 38 can be converted into Equation 40. For this purpose, we define function $g(\eta)$ as follows:

$$
g(\eta)=S_{r c}\left(f_{\tau}, \eta\right) \times \exp \left[j\left(\frac{2 \pi}{c} \cdot \frac{\left(V-v_{a}\right)^{2}-a_{c} R_{B}}{R_{B}} \frac{f_{c}^{2}}{\left(f_{c}+f_{\tau}\right)} \eta^{2}\right)\right]
$$

According to Figure 6, the result of multiplying $\exp \left(-j \frac{\pi^{2}}{\alpha} \eta^{2}\right)$ by $g(\eta)$ should be $S_{r c}\left(f_{\tau}, \eta\right)$. Thus, we have:

$$
\exp \left[-j\left(\frac{2 \pi}{c} \cdot \frac{\left(V-v_{a}\right)^{2}-a_{c} R_{B}}{R_{B}} \frac{f_{c}^{2}}{\left(f_{c}+f_{\tau}\right)} \eta^{2}\right)\right]=\exp \left(-j \frac{\pi^{2}}{\alpha} \eta^{2}\right)
$$

From the above equation, we can derive the following value for $\alpha$ :

$$
\alpha=\frac{c \pi}{2} \frac{\left(f_{c}+f_{\tau}\right)}{f_{c}^{2}} \frac{R_{B}}{\left(V-v_{a}\right)^{2}-a_{c} R_{B}}
$$

On the other hand, in order to set the value of output signal as $g\left(-\frac{\alpha \eta}{\gamma}\right)$ in the keystone process, we derive the following relation:

$$
\frac{f_{c}}{f_{c}+f_{\tau}}=-\frac{\alpha}{\gamma}
$$

By having all other parameters, $\gamma$ can be calculated from the above equation, and finally, $\beta$ can be extracted from the equation $\alpha+\gamma=\beta$.

\section{Moving Target Image Formation Simulation and the obtained Results}

In this section, the simulation results of $\mathrm{RDA}^{15}$ are firstly shown under the condition where OFDM signal is used for a stationary point target. Then the results are shown for the moving target. The values of the important parameters used in this simulation are presented in Table 1.

Table 1. Important Parameters of OFDM SAR System

\begin{tabular}{|c|c|}
\hline Parameter & Value \\
\hline Central Frequency (Centroid) $\left(f_{0}\right)$ & $5.4 \mathrm{GHz}$ \\
\hline (BW) OFDM Signal Bandwidth & $150 \mathrm{MHz}$ \\
\hline Quantity of Subcarrier (N) & 256 \\
\hline Pulse Repetition Frequency (PRF) & $2100 \mathrm{~Hz}$ \\
\hline Velocity of Platform & $200 \mathrm{~m} / \mathrm{s}$ \\
\hline Height & $100 \mathrm{~m}$ \\
\hline Angle of Radiation & $50^{\circ}$ \\
\hline
\end{tabular}

The simulation results in this case are shown in Figure 7 under the condition that all OFDM signal subcarriers are used. The simulation phases include compression in range, RCMC, and azimuth, which have also been illuminated by a related research [11]. 


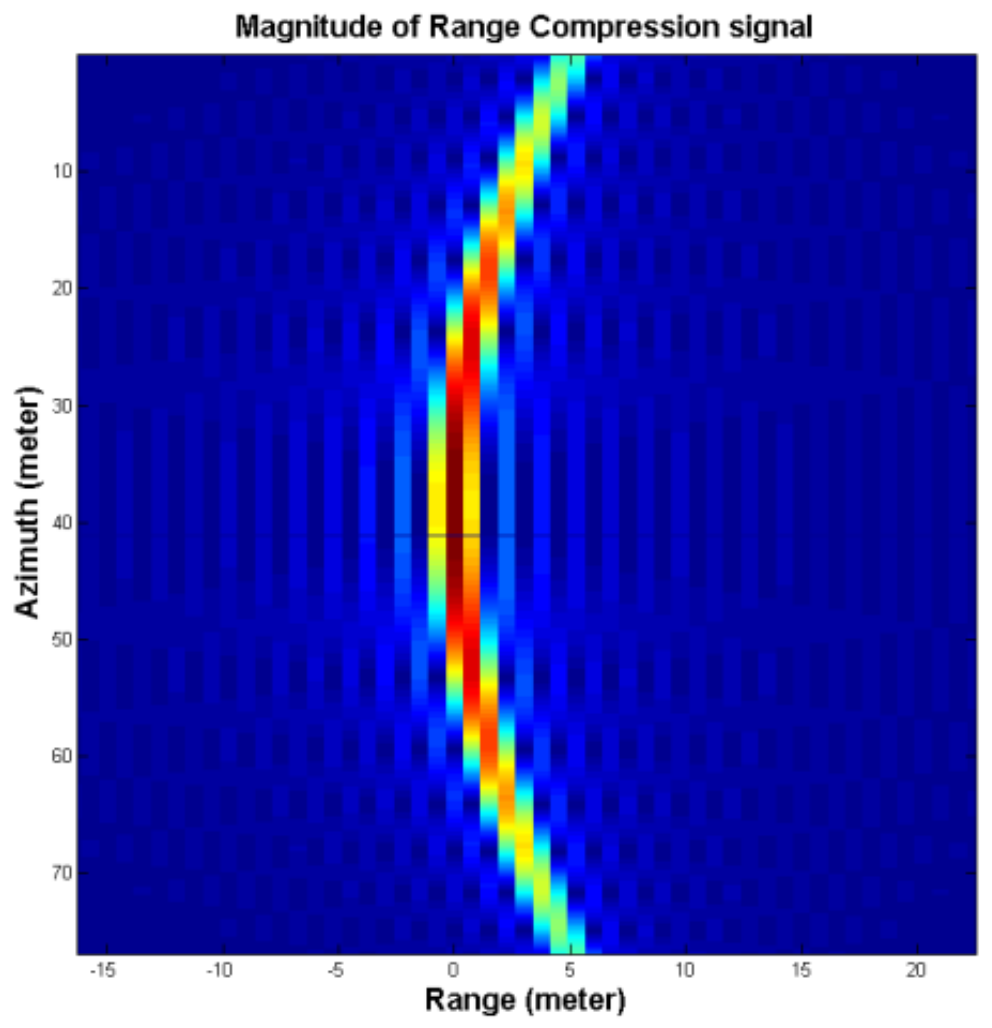

(a)

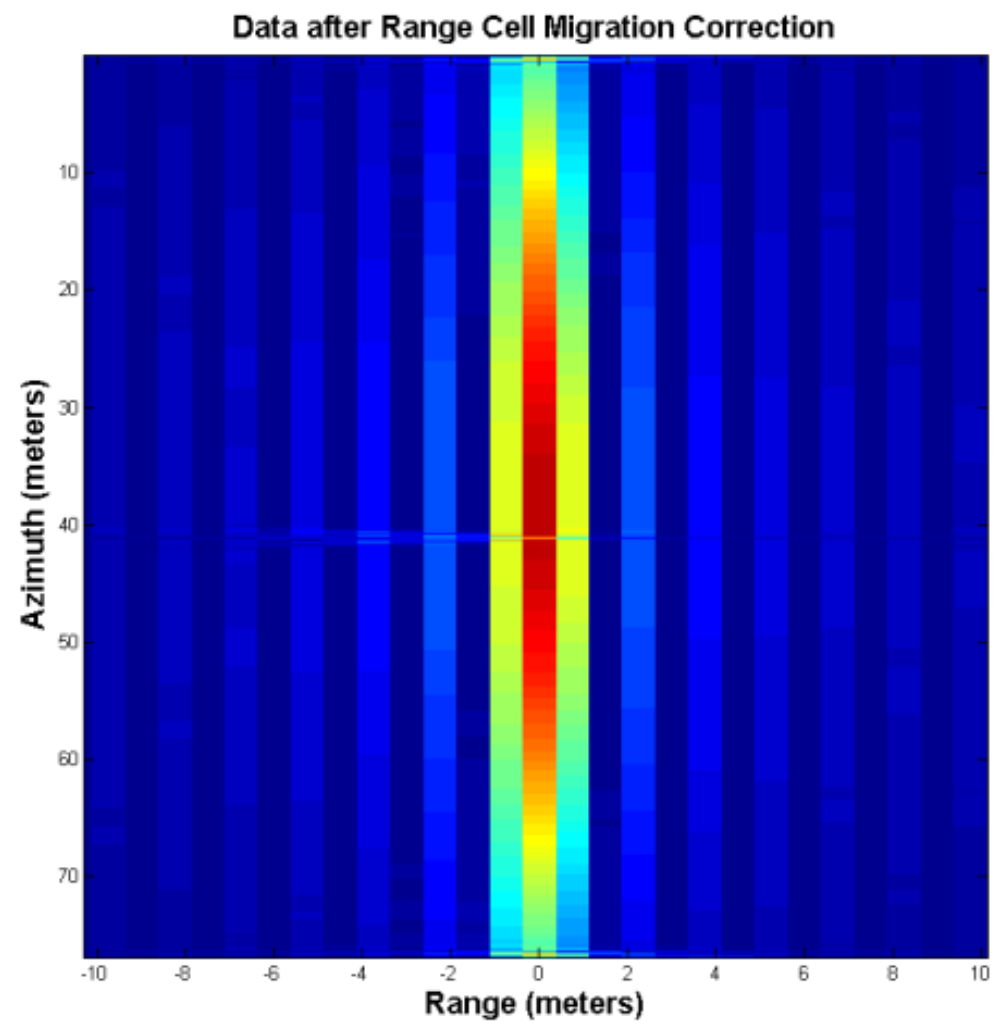

(b) 


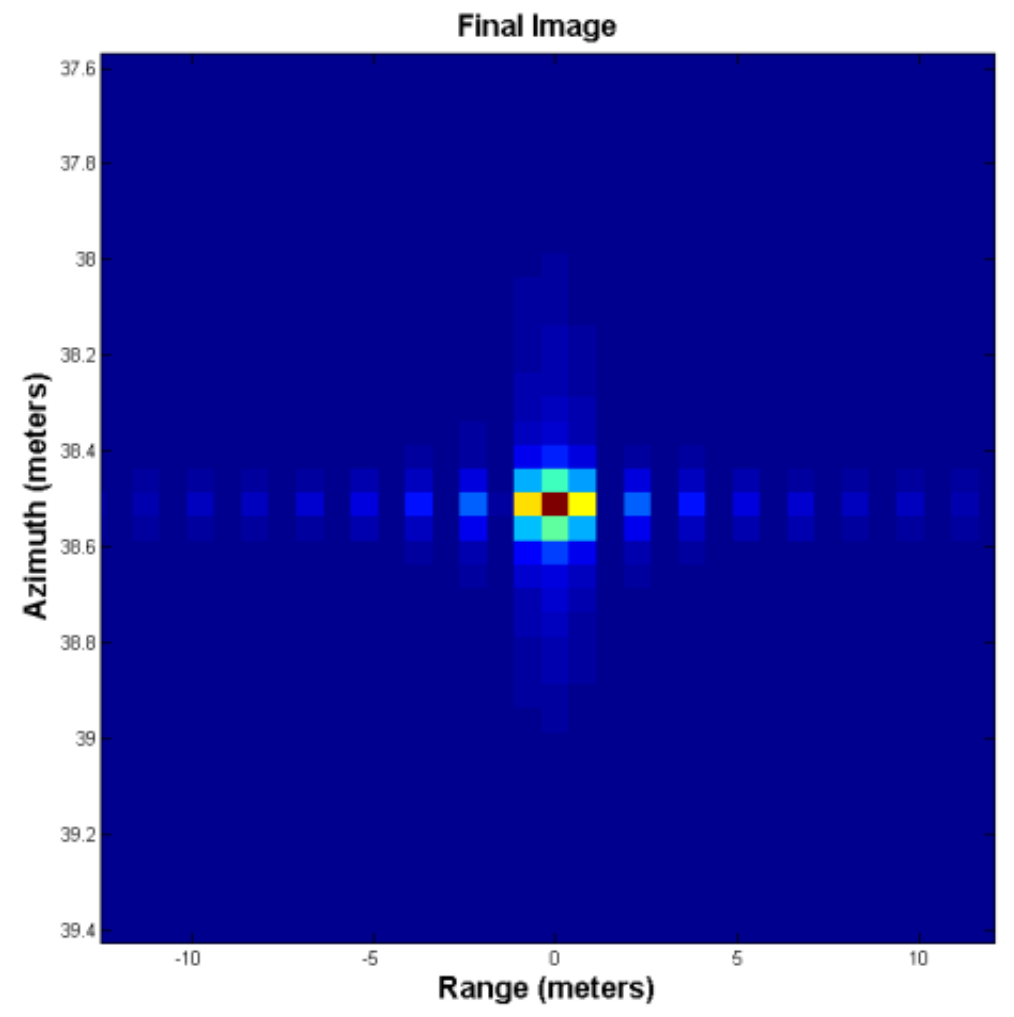

(c)

Figure 7. Simulation of a Stationary Point Target Using All OFDM Subcarriers at the Common Mode of RDA Algorithm

Similarly, if half of the total OFDM subcarriers are randomly used as the transmitted waveform, it will have no destructive effect on the final resolution of the target and the system will be resistant against radar deception (or jamming). The results of this simulation mode are presented in Figure 8. Since the random model with turn-on and turn-off modes for subcarriers may vary in each pulse period and only SAR receiver is informed of this model, the radar deception (jamming) system cannot efficiently match any jamming in the OFDM SAR system. 


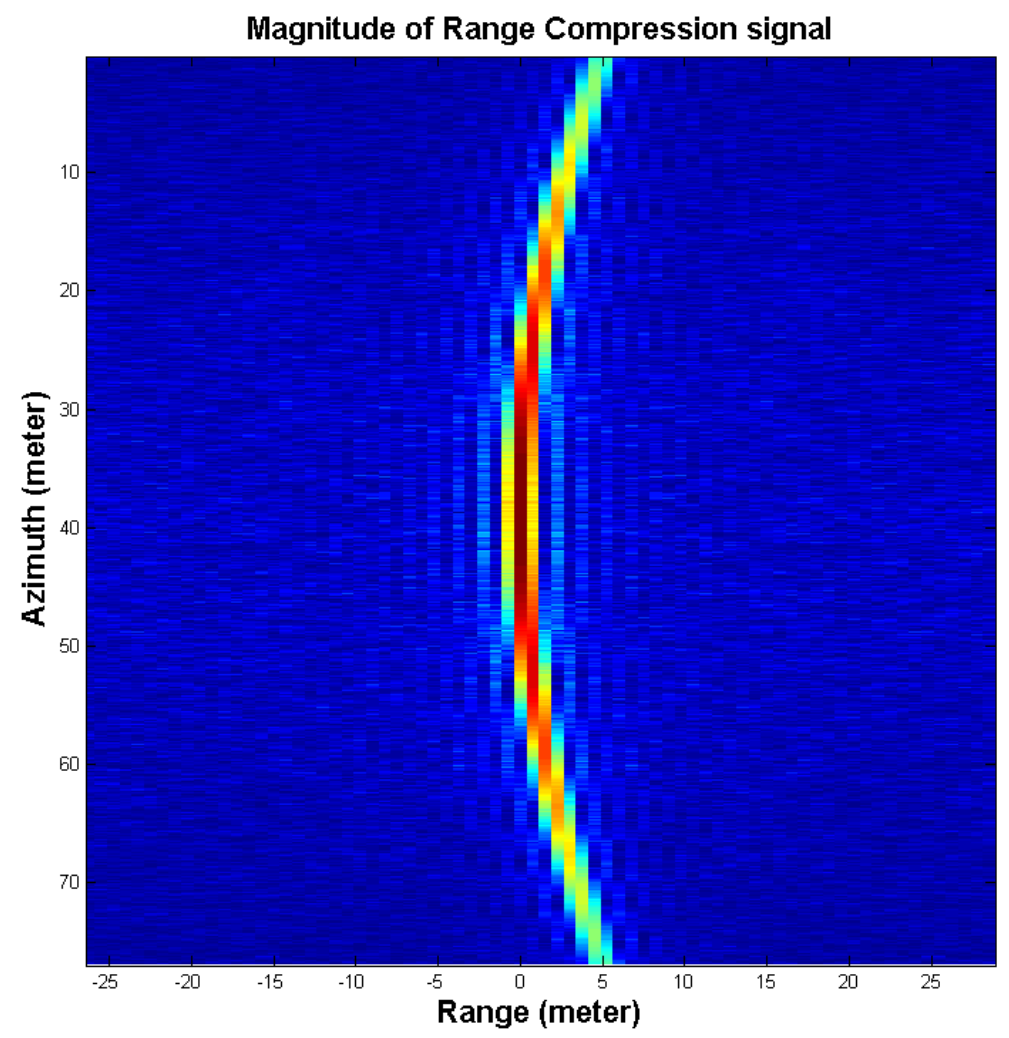

(a)

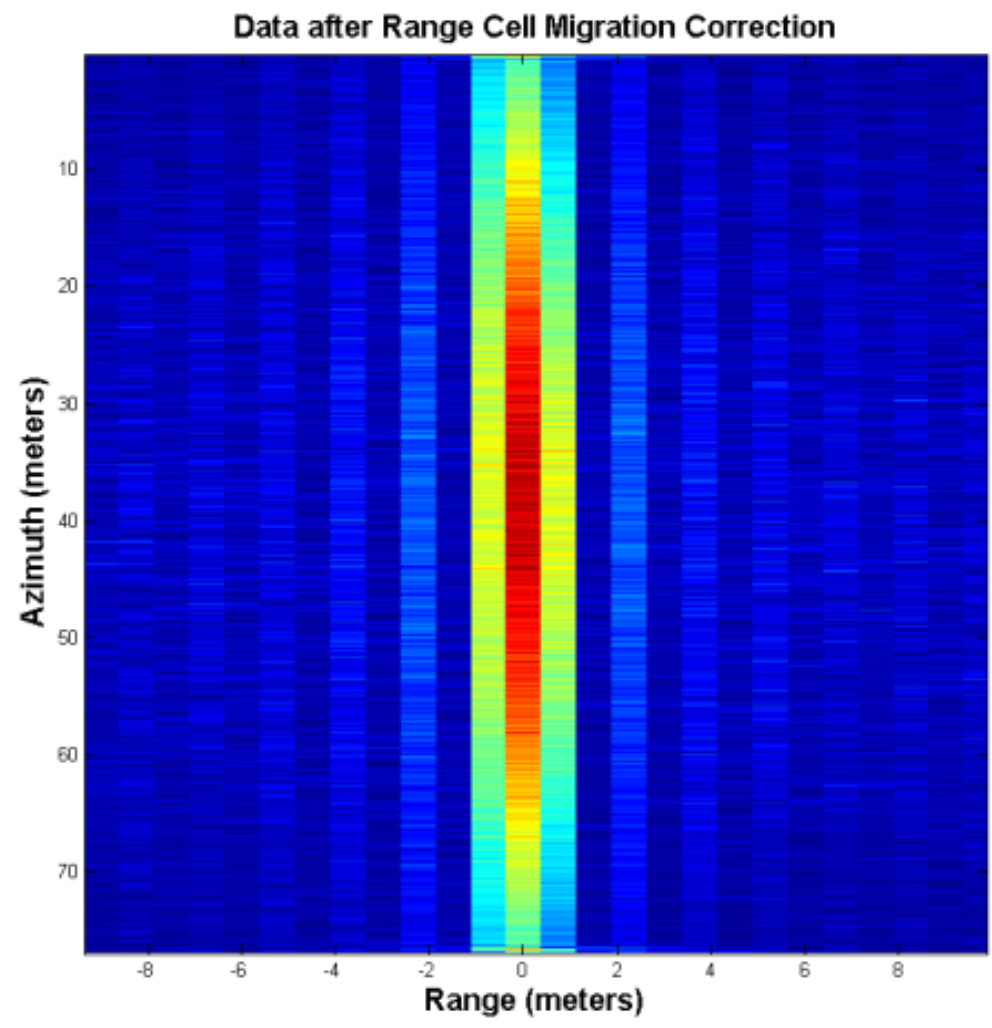

(b) 


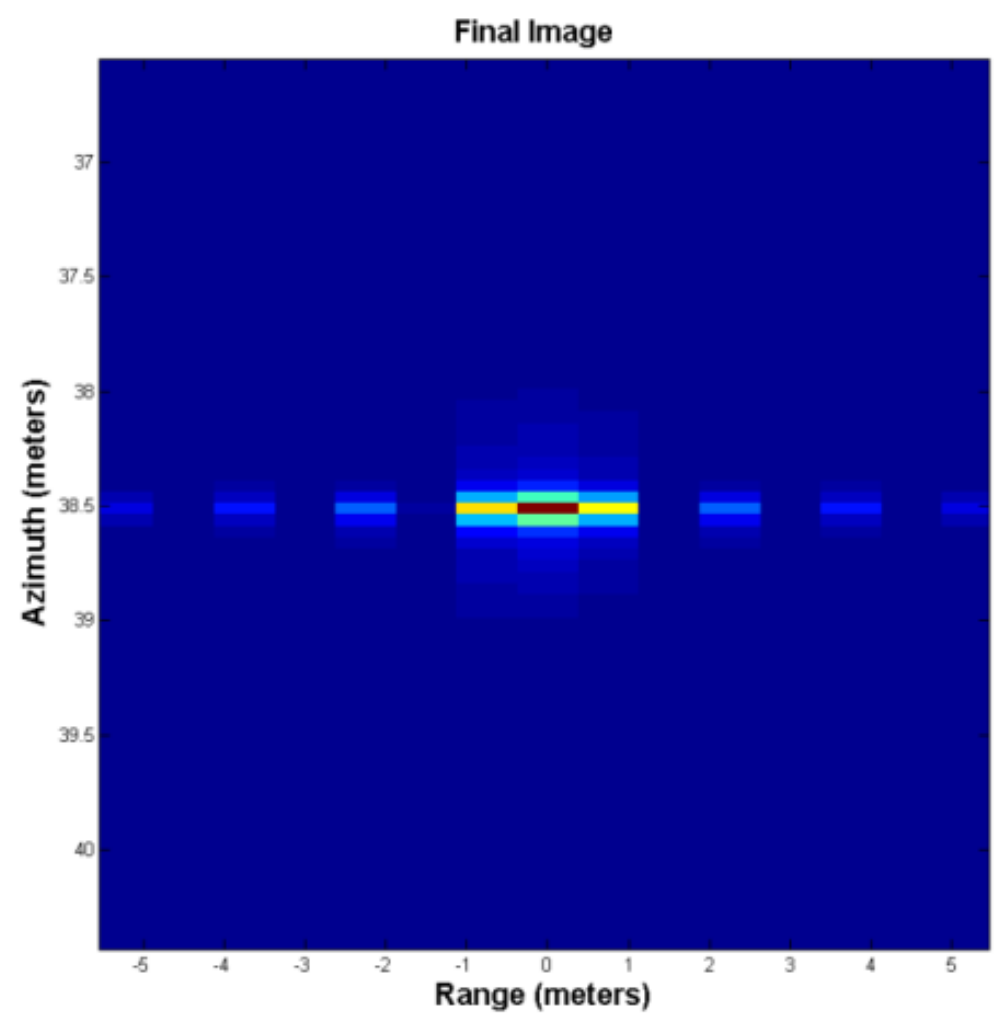

(c)

Figure 8. Simulation of a Stationary Point Target in the Mode of Using Half of OFDM Subcarriers with Random Selection

As mentioned earlier, the common RDA algorithm is not efficient enough for moving targets in the imaging zone. For this purpose, the simulations are performed for a moving point target and its effect is displayed on the resulting image. The target parameters used in the simulation are tabulated below.

Table 2. Moving Parameters of a Point Target

\begin{tabular}{|c|c|}
\hline Parameter & Value \\
\hline Velocity along the platform moving axis & $8 \mathrm{~m} / \mathrm{s}$ \\
\hline Velocity perpendicular to that axis & $5 \mathrm{~m} / \mathrm{s}$ \\
\hline Acceleration along the platform moving axis & $0.1 \mathrm{~m} / \mathrm{s}^{2}$ \\
\hline Acceleration perpendicular to that moving axis & $0.1 \mathrm{~m} / \mathrm{s}^{2}$ \\
\hline
\end{tabular}

In Figure 9 (a), the image is displayed after range compression. In Figure 9 (b), the keystone transform is applied. Figure 9 (c) shows the keystone transform in the range-Doppler domain.

Likewise, Figure 10 (a) shows the image after Doppler centroid correction for a point target. The process Range Curvature correction has been displayed in Figure 10 (b), and the final image indicates the period after compression in azimuth (range) in Figure 10 (c). 


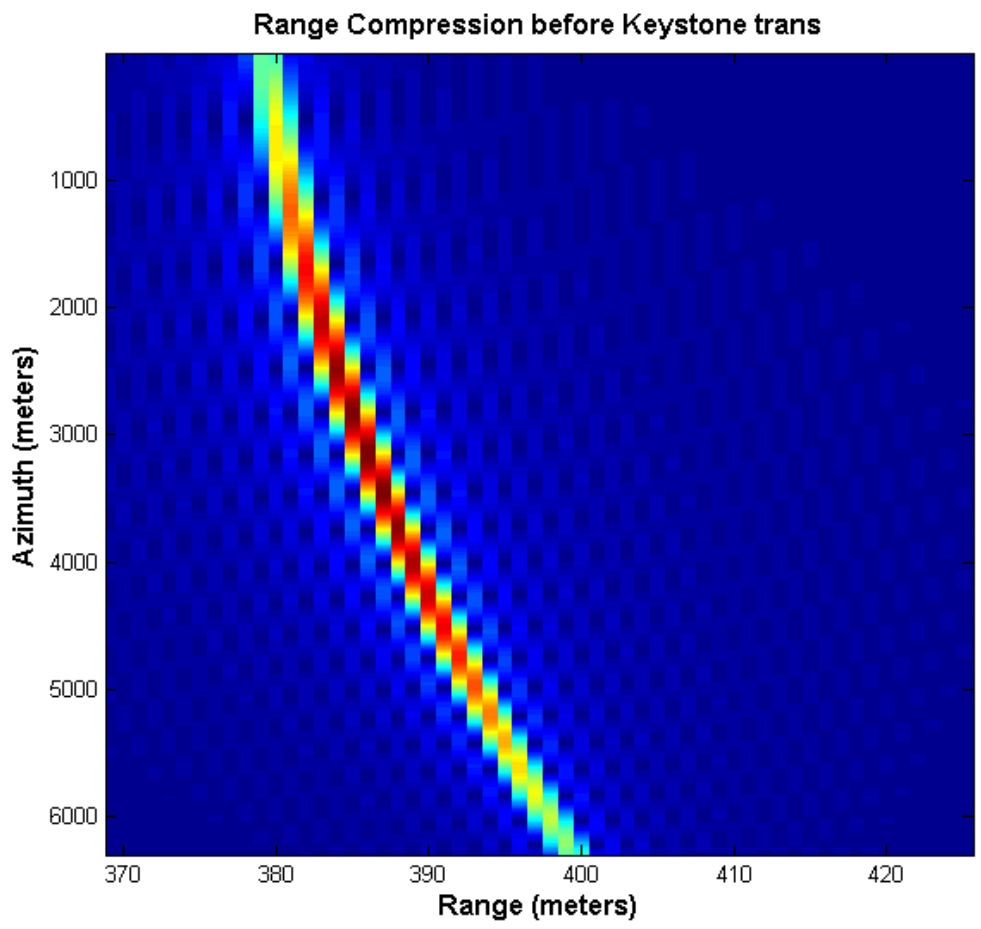

(a)

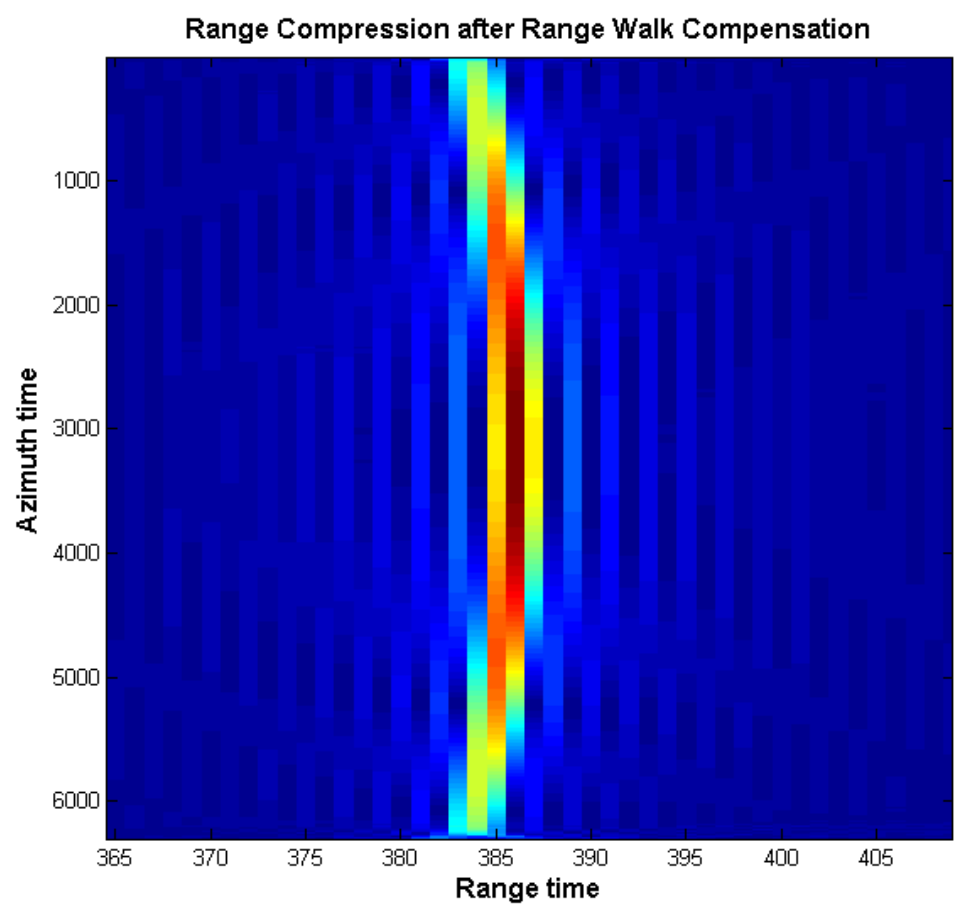

(b) 


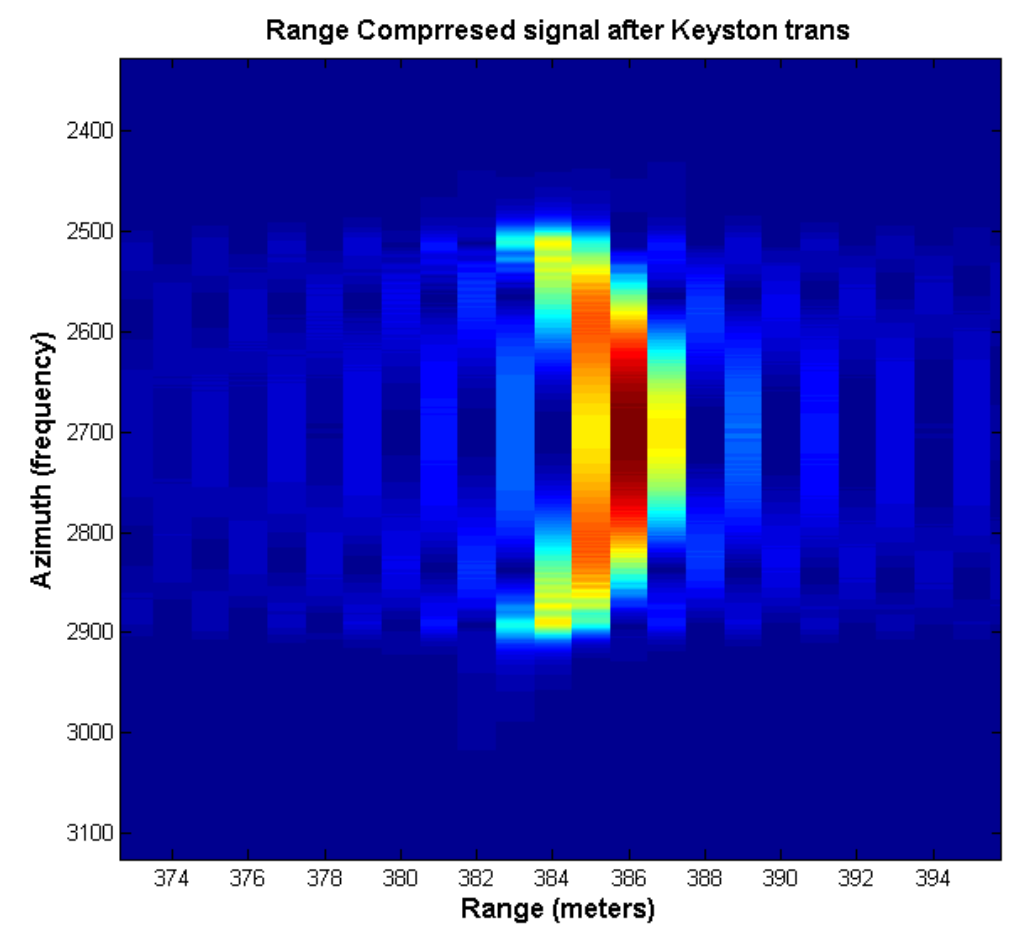

(c)

Figure 9. Demonstration of Range Walk Correction Process, a) Image after Compression in Range, b) Image after Range Walk Compensation , and c) Image after Keystone Transform in the Doppler- Range Field

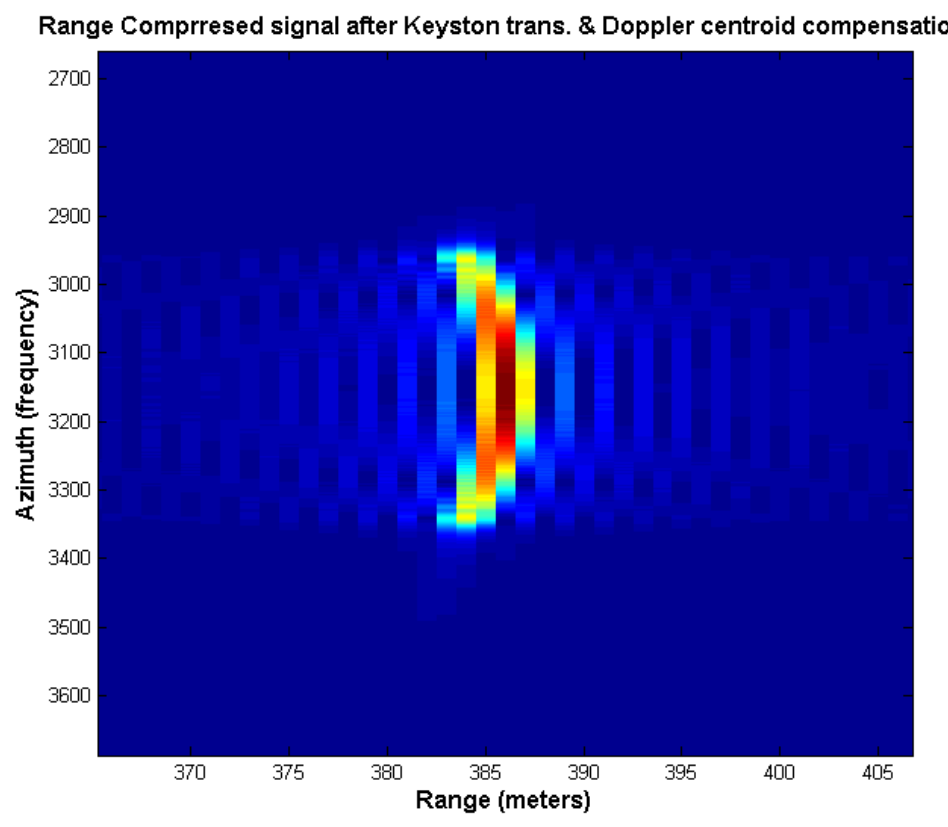

(a) 


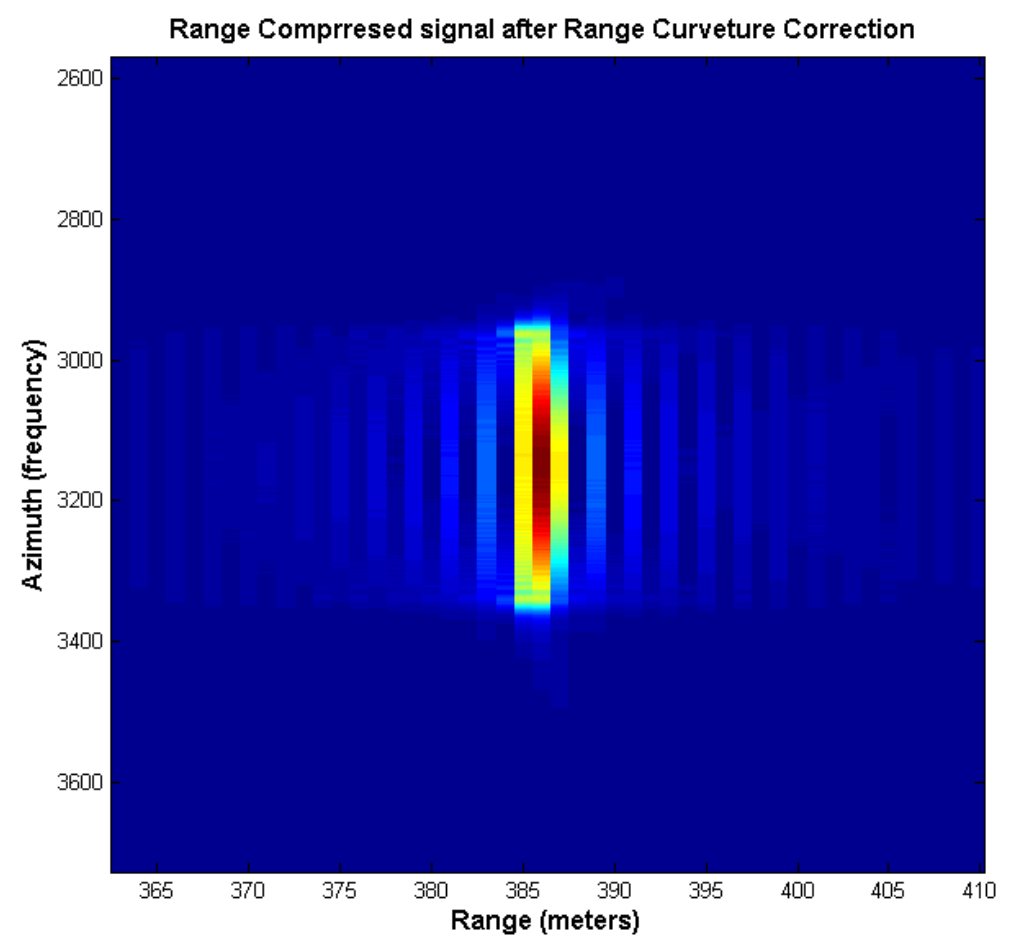

(b)

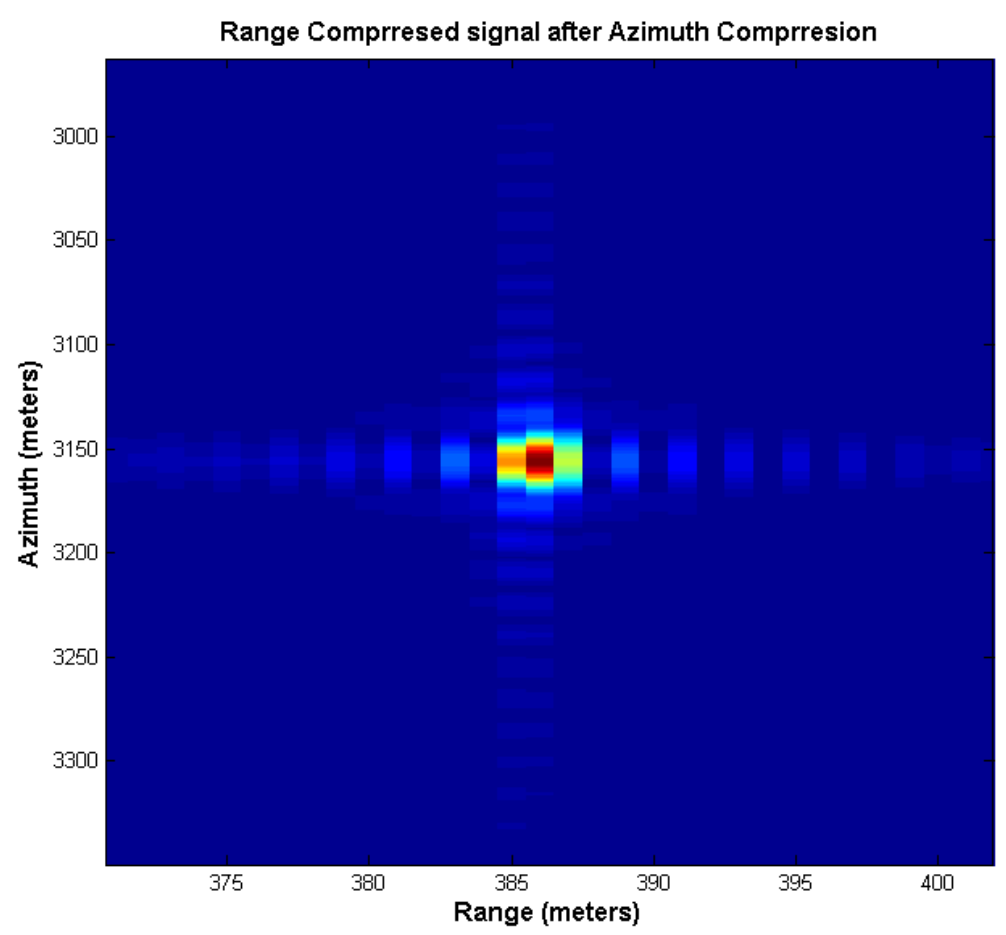

(c)

Figure 10. Demonstration of Range Curvature Correction Process, a) Image After Doppler Centroid Correction, b) Image After Range Curvature Correction, and c) Image After Compression in Azimuth 


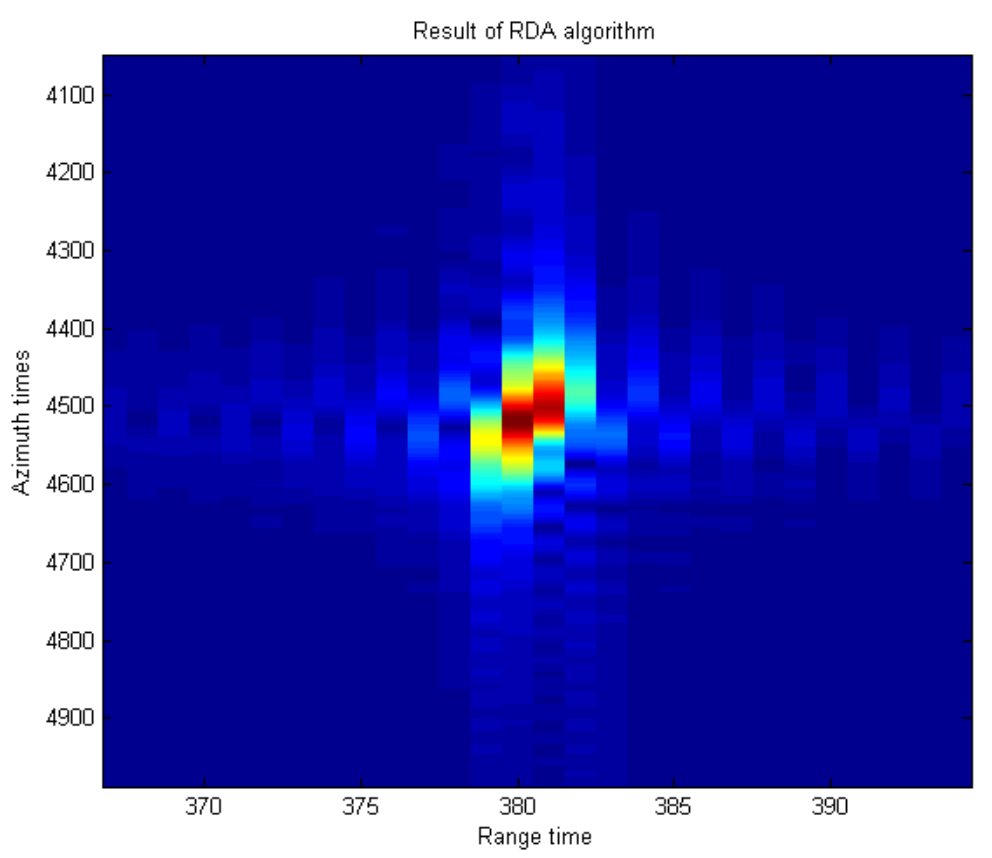

Figure 11. Demonstration of Final Image after applying conventional RDA. The linear component of the range migration significantly blurred the moving target in the SAR image

In Figure 11, the final image of the moving target is displayed using RDA. By exiting the moving target in the image, the RCM curvature rotates around the non-moving target, and the RDA does not compensate the RCM curvature rotation. The first-order keystone transform compensates the effect of the Range Walk moving target.

As seen before, on using the RDA algorithm, the effect of Range Walk and Range Curvation, rotation due from moving target will not compensate and the final image would not be compressed in Range and azimuth, thus blur effect in the image will be displayed.

\section{Conclusions}

In this paper, we purposed a new technique for imaging in SAR by means of OFDM waveform. In this regard, OFDM waveform basics were first reviewed within the context of digital telecommunication systems and the method of producing this signal was presented. Then, by means of a radar-oriented approach, the process of imaging in the range and azimuth of an SAR system was presented within the framework of RDA. When a target moves with a certain velocity and acceleration within the imaging zone, the custom algorithms are not usually efficient, and the acquired image becomes opaque and unclear. Noting this problem, we proposed an algorithm for imaging moving targets based on OFDM waveform and first-order keystone transform. The simulation results indicated the efficiency of the algorithm versus the quality of the generated images.

The results of the simulation show that the first-order keystone transform is efficient in presenting a moving target in comparison to RDA.
In the usual RDA method, due to the inability to compensate Range Walk and Range Curvation and rotation due to moving target, the final image will be unclear. However, the first-order keystone transform will compensate all the effects of moving target in the image and also estimate the moving target parameters.

\section{REFERENCES}

[1] R. O. Harger. "Synthetic Aperture Radar Systems: Theory and Design". Academic Press, New York,1970.

[2] Fazel, K. and Kaiser, S., "Multi-Carrier and Spread Spectrum Systems: From OFDM and MC-CDMA to LTE and WiMAX", 2nd Edition, John Wiley \& Sons, 2008.

[3] Bahai, A. R. S., Saltzberg, B. R. and Ergen, M., "Multi Carrier Digital Communications: Theory and Applications of OFDM", Springer, 2004.

[4] deCouasnon, T., Monnier, R. and Rault, J.B., "OFDM for digital TV broadcasting, Signal processing", (ELSEVIER), 39, 1994, 1-32.

[5] Garmatyuk. D, Brenneman. M, "Adaptive Multicarrier OFDM SAR Signal Processing "IEEE TRANSACTIONS ON GEOSCIENCE AND REMOTE SENSING, VOL. 49, NO. 10, OCTOBER 2011

[6] Garmatyuk. D, Jonathan. S Kauffman. K, "Multifunctional Software-Defined Radar Sensor and Data Communication System", IEEE SENSORS JOURNAL, VOL. 11, NO. 1, JANUARY 2011

[7] W. Wang, "Space-Time Coding MIMO-OFDM SAR for High-Resolution Imaging", IEEE TRANSACTIONS ON 
GEOSCIENCE AND REMOTE SENSING, VOL. 49, NO. 8, AUGUST 2011

[8] F.Zhou, R. Wu, M. Xing and Z. Bao, "Approach for single channel SAR ground moving target imaging and motion parameter estimation", IET Radar Sonar Navig., 2007, 1, (1), pp. 59-66

[9] Daiyin Zhu, Yong Li, "A Keystone Transform without Interpolation for SAR Ground Moving-Target Imaging", IEEE GEOSCIENCE AND REMOTE SENSING LETTERS, VOL. 4, NO. 1, JANUARY 2007

[10] Yang Jungang, "New Approach for SAR Imaging of Ground Moving Targets Based on a Keystone Transform", IEEE GEOSCIENCE AND REMOTE SENSING LETTERS, VOL. 8, NO. 4, JULY 2011

[11] Cumming, I.G., Wong, F.H.: "Digital Processing of Synthetic Aperture Radar Data". Chapter 4, Artech House, Norwood, 2005

[12] Soumekh, M., "Synthetic Aperture Radar Signal Processing", New York, John Wiley\& Sons, 1999.

[13] Levanon, N. and Mozeson, E., "Radar Signals", New Jersey, John Wiley \& Sons, 2004, 57-66.

[14] Weiss, L.G., "Wavelets and Wideband Correlation Processing", IEEE signal Processing Magazine, Jan. 1994, 13-32.

[15] M. Soumekh, "SAR-ECCM using phase-perturbed LFM chirp signals and DRFM repeat jammer penalization", IEEE Trans. Aerospace and Electronic Systems, vol. 42, no. 1, pp. 191-205, Jan. 2006. 